\title{
Common Ophthalmic Preservatives in Soft Contact Lens Care Products: Benefits, Complications, and a Comparison to Non-Preserved Solutions
}

\author{
Chelsea S Bradley $\mathbb{D}^{\prime}$ \\ Lindsay A Sicks (D) \\ Andrew D Pucker (iD) ${ }^{2}$ \\ 'Illinois College of Optometry, Chicago, \\ IL, USA; ${ }^{2}$ School of Optometry, \\ University of Alabama at Birmingham, \\ Birmingham, AL, USA
}

Purpose: Preservatives are essential for preventing contact lens (CL)-related microbial keratitis (MK). The purpose of this review is to summarize the current knowledge related to the use of common ophthalmic preservatives in CL care products with respect to both safety and efficacy.

Methods: Manuscripts written in English were obtained by searching PubMed.gov with the term contact lens plus antimicrobial, benzalkonium chloride, biguanide, Aldox, polyquaternium, preservative, thimerosal, EDTA (ethylenediaminetetraacetic acid), chlorhexidine, or blister pack.

Results: This review found that first-generation preservatives are no longer used in CL multipurpose solutions (MPS) due to their high levels of ocular toxicity. Modern, highmolecular-weight preservatives, including polyquaternium-1 (PQ-1) and biguanides (PHMB), are generally effective against bacteria, minimally effective against fungi, and not effective against Acanthamoeba. PQ-1 and PHMB are likely safe when used with CLs, but they may cause ocular adverse events, with roughly equal risk between the two preservatives. Some CL MPS contain both PQ-1 and PHMB, but no increased risk of adverse events has been reported when combining the two. Hydrogen-peroxide $\left(\mathrm{H}_{2} \mathrm{O}_{2}\right)$ solutions are effective against all common ocular microbes, including Acanthamoeba, and they have been proven safe with proper compliance. Povidone-iodine (P-I) solutions are not currently commercially available in North America, but they have been shown in other countries to be safe and effective.

Conclusion: Patients should be monitored when using PQ-1 or PHMB-containing solutions since they have been associated with ocular adverse events. If events are detected, patients should be switched to an alternative solution. $\mathrm{H}_{2} \mathrm{O}_{2}$ or P-I solutions are preferred for any patient who may expose their CLs to water because they are the only solution categories effective against Acanthamoeba.

Keywords: preservatives, contact lenses, care systems, multipurpose solutions

\section{Introduction}

Microbial keratitis (MK) is a potentially sight-threatening corneal infection, which may be caused by bacteria, fungus, or amoebae. MK is one of the leading causes of blindness worldwide, with its prevalence varying by geographical region. ${ }^{1}$ In the United States, the incidence of MK is about 30,000 cases per year, ${ }^{2}$ while the incidence in India is roughly two million cases per year. ${ }^{3}$ In a 2002 study, Lam et al reported an incidence of $\mathrm{MK}$ in Hong Kong of 0.63 per 10,000 patient years, while the authors found an incidence of 0.36 per 10,000 patient years in Scotland. ${ }^{4}$ The
Correspondence: Andrew D Pucker Email apucker@uab.edu 
incidence and morbidity of $\mathrm{MK}$ is increasing in developing regions, as highlighted above by the statistics in India and by MK being the second leading cause of blindness in Africa just behind cataracts. ${ }^{1}$ While there are many factors such as age and sex that increase the risk of MK, contact lens (CL) wear is a top risk factor, ${ }^{2,5}$ and the frequency of soft CL-related MK has been unfortunately increasing in recent years. ${ }^{3}$ This is highly problematic since $10 \%$ to $14 \%$ of cases result in a loss of two or more lines of bestcorrected visual acuity. ${ }^{5}$

While the difference is small, the risk for MK is lower in patients who wear daily disposable CLs compared to patients who wear reusable CLs, likely due to the fewer care steps involved with daily disposable CLs. ${ }^{3,6}$ Unfortunately, 2019 market research from Contact Lens Spectrum indicates that only about $35 \%$ of all soft CL fits were daily disposable CLs, while reusable soft CLs comprised about $44 \%$ of the CL market. ${ }^{7}$ Considering the risk for $\mathrm{MK}$ is not eliminated with daily disposable CL wear and prescribing trends still favor reusable CLs, it is important to consider the risk for MK in all soft CL wearers.

The increased risk of MK with CL wear, as well as the potential for visual morbidity, requires the use of CL care products with reusable CLs. These products specifically require the ability to reduce or eliminate microbes associated with CL complications. Most CL care products include preservatives as active ingredients to combat these microbes. These products must be highly effective at eliminating a wide array of microbes but also gentle enough for use on the human eye. The field currently lacks a general summary of these versatile products. Therefore, the purpose of this manuscript was to examine the various ophthalmic preservatives as used with CLs in terms of their mechanisms of action, effectivity against common ocular microbes, use in soft CL multi-purpose solutions (MPS), and effects on the ocular surface in conjunction with soft CL wear. Additionally, nonpreserved CL solutions are discussed in comparison to preserved CL MPS. A comprehensive summary of these data will not only allow the field to move forward, but it will allow clinicians to better educate and care for their patients.

\section{Methods}

The intent of this review was to summarize the safety and efficacy of preservatives used in soft CL solutions. Manuscripts were obtained by searching PubMed.gov on or before July 31,2021 , with the term contact lens plus antimicrobial, benzalkonium chloride, biguanide, Aldox, polyquaternium, preservative, thimerosal, ethylenediaminetetraacetic acid (EDTA), chlorhexidine, or blister pack. Recovered texts were searched to find additional references. Meeting abstracts and trial registrations were excluded during the search. No date exclusions were applied, and only articles written in English were considered in this review.

\section{Results}

\section{Preservatives in MPS - Standardizations for Efficacy and Safety}

When evaluating a MPS preservative, one should consider whether it provides effective antimicrobial activity against microbes that commonly cause MK. The International Organization for Standardization (ISO) has outlined criteria for assessing whether a preservative is effective in the following standard: ISO 14729:2001 — Ophthalmic Optics - Contact Lens Care Products Microbiological Requirements and Test Methods for Products and Regimens for Hygienic Management of Contact Lenses. ${ }^{8}$ ISO 14729 states that starting concentrations of bacteria in phosphate-buffered saline in a test tube must be reduced by no less than $3 \log _{10}(99.9 \%)$ and fungi by no less than $1 \log _{10}(90 \%)$ cells while soaking within the manufacturer's recommended disinfection time (no rubbing or rinsing necessary). If the solution cannot meet this criterion, then completion of the entire recommended regimen outlined in the package insert (with rubbing and rinsing steps) can be used to assess for reduction in numbers of microbes adherent to CLs to less than or equal to 10 colony-forming units. ${ }^{8,9}$

The United States Food and Drug Administration (FDA) set standards that preceded ISO standards for the reduction of bacteria. The FDA requires the D-value (the calculated time required to reduce a population of microorganisms by $90 \%$ or $1 \log _{10}$ ) to be calculated, although a standard value was never set. The FDA also requires no survivors be present after a lens is treated according to manufacturer instructions when tested by the Microbial Challenge Multi-Item Test. ${ }^{9}$ However, French, British, and Dutch authorities each specified different guidelines, creating a cumbersome process for manufacturers who need their MPS approved in each country. Thus, the ISO standards were set for continuity and simplicity and are more commonly used than the FDA guidelines. ${ }^{9}$ For the remainder of this review, "effective" will refer to bacterial 
load reduction as standardized by the ISO, unless otherwise noted.

ISO 14729 also outlines the specific strains of bacteria, mold, and fungi that MPS need to be tested against: Serratia marcescens American Type Culture Collection (ATCC) 13880, Pseudomonas aeruginosa ATCC 9027, Staphylococcus aureus ATCC 6538, Candida albicans ATCC 10231, and Fusarium solani ATCC 36031. However, the standard does not require solutions be tested against Acanthamoeba due to the lack of standard methodology for testing solution efficacy, the lack of standard recovery and enumeration protocols, the lack of standard strains, and the rare incidence of Acanthamoeba keratitis. $^{9,10}$ ISO considers the reduction in bacteria in a CL lens case as an adequate measure for the prevention of Acanthamoeba by limiting bacteria as a food source for the organism. ${ }^{10}$ The lack of standardization must be considered when evaluating the validity of any study testing for MPS efficacy against Acanthamoeba.

Additionally, there are questions surrounding whether and how conclusions from studies based on ISO standards may be applied clinically. This is largely due to the difference in in vitro versus in vivo environments. Studies have found that the efficacy of MPS against ISO standard strains may differ in the presence of organic soil. ${ }^{11-13}$ Organic soil found in the tear film may be transferred from a removed CL into a MPS and may neutralize the disinfecting agent. ${ }^{9}$ The FDA standards require proof of efficacy against a laboratoryprepared organic soil composed of a mixture of killed Saccharomyces cerevisiae and inactivated fetal bovine serum. However, some argue testing against organic soil is useless considering the FDA standard protocol does not accurately represent tear film components, soil varies in the tear film from patient to patient, and soil is largely removed by the rubbing and rinsing step required of most MPS. ${ }^{37}$ Therefore, ISO standards do not require a test including organic soil. ${ }^{9}$ Additionally, studies have compared ISO standard strains to clinical isolates, ${ }^{14-16}$ and investigators have found the efficacy of MPS may be reduced against clinical isolates (outlined in Modern High Molecular Weight Preservatives).

While ISO standards outline appropriate test strains and standards for efficacy, standards for study design are sometimes vague. For example, ISO standardizes microbial growing conditions, including growth time, temperature, and nutrients, but it does not specify whether to use glass or plastic test tubes. This is problematic considering certain preservatives in MPS are known to adhere to either plastic (polyquaternium-1 (PQ-1)) ${ }^{10}$ or glass (biguanides (PHMB)). ${ }^{15}$ Additionally, some wording in ISO 14729 is ambiguous, such as allowing for researchers to define some testing conditions on their own. ${ }^{9}$ These ambiguities and researchers' choices of organism strain, solution formulation, and organism recovery lead to varying findings, even amongst studies using identical methodology. ${ }^{10}$ Such variations are important to remember when evaluating studies for clinical relevance.

Once a preservative has been proven effective against the standard microbes, the safety profile also needs to be proven to gain FDA or other international standards approval. CL care products are in a class that requires a $510(\mathrm{k})$, or "Premarket Approval." Briefly, this requires proving a new CL product is "substantially equivalent" in safety and efficacy to similar commercially available products. This includes microbiology and chemistry testing for efficacy, as summarized above, though this is often abandoned in favor of ISO standards. The FDA $510(\mathrm{k})$ standards are still used for toxicology and clinical testing proving safety. Recommendations for minimum toxicology testing include in vitro tests and in vivo acute ocular irritation tests as defined by the United States Pharmacopeia (USP)/National Formulary (NF) 23 and XXII, respectively. However, the FDA stipulates that the ocular irritation test "should not be needed in cases where formulations contain known ocular irritants," which may apply to many preservatives and other ingredients found in MPS. Instead, the FDA requires manufacturers to indicate on the label that the product contains an ocular irritant. The recommendations also state that any test suggested in this toxicology section may be replaced by a suitable alternative if the alternative test has been validated or can be justified for use as an alternative, ${ }^{17}$ which may lead to difficulty when comparing studies.

Clinical recommendations include recording adverse reaction data, slit-lamp findings, symptoms/problems/complaints, visual acuities, and discontinuations. An adverse reaction is defined as "a hazardous, sight-threatening condition," and findings must be photographed or detailed in drawings for submission of the $510(\mathrm{k})$ application. Pertinent slit-lamp findings include corneal epithelial edema, corneal epithelial microcysts, corneal stromal edema, corneal neovascularization, corneal staining, bulbar conjunctival hyperemia, palpebral conjunctival findings, and other (eg, infiltrates). All these findings are graded on a scale of 0 to $4(0=$ none, $1=$ trace, $2=$ mild, 3 = moderate, $4=$ severe), with any findings graded 
2 or higher requiring further explanation in the $510(\mathrm{k})$ application. Symptoms generally include reports of comfort, ease of handling, and vision, and are graded on a 0 to 5 scale $(0=$ excellent, $1=$ very good, $2=$ good, $3=$ poor, 4 $=$ very poor, $5=$ unacceptable), but the $510(\mathrm{k})$ standards do not define differences between acceptable and unacceptable grades. ${ }^{17}$ Interestingly, some studies outlined later in this review do not use these recommendations as their safety standards; rather, the authors define their own clinical grading scales. This likely explains many betweenstudy safety differences.

In summary, an ideal preservative is effective at low concentrations and safe at high concentrations. A preservative that meets both of these standards has a wide "margin of safety." 18 Achieving both requirements can prove difficult, as a balance is needed between low ocular surface toxicity and high antimicrobial efficacy. The ability of various MPS to achieve both requirements is outlined below.

\section{Preservatives Used in Soft CL MPS - Mechanisms of Action, Safety, and Efficacy}

This section will summarize the literature regarding the mechanism of action, safety, and efficacy of each common preservative used in commercially available CL MPS. It is important to consider both how effective each preservative is, as well as how each may affect the ocular surface when used as directed by the manufacturer. Specific MPS will not be named in this literature review, but the preservatives found in each commercially available or previously available MPS detected in the literature are outlined in Table 1.

\section{First Generation Preservatives}

Mercury-based thimerosal is one of the oldest preservatives used in CL MPS. The mechanism of action of thimerosal is complicated and concentration-dependent, but it primarily induces antimicrobial action by disrupting calcium influx into cells. ${ }^{19}$ Thimerosal has a well-documented history of being effective against ocular-pathogenic bacteria and fungal species compared to chlorhexidine and PHMB-based solutions. ${ }^{20}$ It has been shown to be ineffective against Acanthamoeba, unless combined with other antimicrobials such as EDTA ${ }^{21,22}$ or chlorhexidine. ${ }^{23}$

While thimerosal's mechanism of action allows it to be exceptionally effective against both gram-positive and gram-negative bacteria, its small molecule size allows it to be easily absorbed and released by CLs. Thimerosal is then leached from CLs onto the ocular surface, and has been commonly associated with corneal irritation, stinging, conjunctival hyperemia, limbal epithelial changes, corneal infiltrates, palpebral lid changes, and corneal staining after CL wear. ${ }^{24}$ Two investigations reported unexplained epitheliopathy ${ }^{25,26}$ and conjunctivitis ${ }^{25}$ amongst $\mathrm{CL}$ wearers. These adverse events were confirmed to be from thimerosal in MPS, as they resolved upon discontinuation of the product and recurred when the ocular surface and/or skin was challenged with isolated thimerosal. ${ }^{25}$

As the clinical complications are often delayed in time from onset of use, ${ }^{25,27}$ thimerosal-induced anterior segment pathology is likely an immune-driven delayed hypersensitivity reaction rather than a direct irritant. ${ }^{25,27,28}$ Studies report a roughly $5 \%$ to $8 \%$ incidence of thimerosal-related delayed hypersensitivity in the United States, ${ }^{28-30}$ confirming immune response as the most common reason for the negative effects. However, thimerosal has also been shown to induce an immediate toxic response both in vitro via inhibition of mitotic activity in human corneal epithelial cells ${ }^{31}$ and in vivo, ${ }^{32}$ indicating that it can also act as a direct ocular irritant. In addition to ocular adverse events, thimerosal is known to cause dermatological delayed hypersensitivity reactions. Sertoli et al reported contact dermatitis on the hands of patients who cleaned their CLs with thimerosal-preserved MPS. ${ }^{29}$ The authors confirmed this reaction was due to thimerosal via a re-challenge patch test that isolated thimerosal from MPS that also contained chlorhexidine and BAK, and found that thimerosal was the only preservative of the three that elicited a positive skin reaction. ${ }^{29}$ A later study confirmed potential skin issues with thimerosal when they showed a high reaction rate with skin patch testing of thimerosal isolated from MPS, but not with other isolated chemicals found in MPS that contain thimerosal. ${ }^{25}$ These dermatological findings raised concern for similar thimerosal-induced irritation on the skin around the eyelids, but the literature does not specifically report any eyelid skin reactions to thimerosal MPS.

With toxicity data mounting (immuno-allergic ocular surface changes, dermatological reactions, and keratoconjunctivitis), Rietschel and Wilson urged the FDA in 1982 to find alternative means for CL disinfection that were similarly affordable as thimerosal. Thimerosal MPS products began to be withdrawn from the market in the late $1980 \mathrm{~s}^{32}$ Thimerosal is no longer used in commercially available MPS because of these safety issues.

Chlorhexidine is a less commonly used bacteriostatic molecule, ${ }^{28}$ that causes cell membrane disruption ${ }^{33}$ and is 
Table I Contact Lens Multipurpose Solutions Detected in the Literature

\begin{tabular}{|c|c|c|}
\hline Manufacturer & Solution Name & Preservative(s) \\
\hline \multirow[t]{15}{*}{ Alcon } & OptiFree PureMoist & Polyquad (polyquaternium $0.001 \%$ ) \\
\hline & & Aldox (myristamidopropyldimethylamine $0.0005 \%$ ) \\
\hline & OptiFree Replenish & Polyquad (polyquaternium $0.001 \%$ ) \\
\hline & & Aldox (myristamidopropyldimethylamine $0.0005 \%$ ) \\
\hline & OptiFree Express & Polyquad (polyquaternium $0.001 \%$ ) \\
\hline & & Aldox (myristamidopropyldimethylamine $0.0005 \%$ ) \\
\hline & Opti-Soft* & Polyquad (polyquaternium $0.001 \%$ ) \\
\hline & ClearCare/ClearCare with HydraGlyde & Non-preserved \\
\hline & & Sterile micro-filtered hydrogen peroxide $3 \%$ \\
\hline & Soac-Lens* & Thimerosal $0.004 \%$ \\
\hline & Preflex* & Thimerosal $0.004 \%$ \\
\hline & Normol* & Thimerosal $0.001 \%$ \\
\hline & & Chlorhexidine gluconate $0.005 \%$ \\
\hline & FlexCare Rinsing \& Storage Solution* & Thimerosal $0.001 \%$ \\
\hline & & Chlorhexidine gluconate $0.005 \%$ \\
\hline \multirow[t]{4}{*}{ AMO (Johnson \& Johnson Vision) } & Acuvue RevitaLens & Alexidine (alexidine dihydrochloride $0.00016 \%$ ) \\
\hline & & Polyquad (polyquaternium-I $0.0003 \%$ ) \\
\hline & Complete Moisture (Comfort) Plus MPS & Polyhexamethylene biguanide $0.0001 \%$ \\
\hline & Oxysept UltraCare & $\begin{array}{l}\text { Non-preserved } \\
\text { Sterile 3\% hydrogen peroxide }\end{array}$ \\
\hline \multirow[t]{9}{*}{ Bausch + Lomb } & Renu Advanced Formula & Polyhexamethylene biguanide $0.0001 \%$ \\
\hline & & Polyquaternium-I $0.001 \%$ \\
\hline & & Alexidine (alexidine dihydrochloride $0.00016 \%$ ) \\
\hline & Renu Multiplus & DYMED $®$ (polyaminopropyl biguanide $0.0001 \%$ ) \\
\hline & Renu MoistureLoc & Alexidine $0.00045 \%$ \\
\hline & Biotrue & Polyaminopropyl biguanide $0.00013 \%$ \\
\hline & & Polyquaternium $0.0001 \%$ \\
\hline & Soflens* & Thimerosal $0.001 \%$ \\
\hline & & Chlorhexidine $0.005 \%$ \\
\hline CooperVision & Refine I-Step & Non-preserved \\
\hline & & Sterile $3 \%$ hydrogen peroxide \\
\hline \multirow[t]{3}{*}{ CIBA Vision } & SOLOCare Plus & Polyhexamethylene biguanide $0.0001 \%$ \\
\hline & AQuify* & Polyhexamethylene biguanide $0.0001 \%$ \\
\hline & AOSEPT & $\begin{array}{l}\text { Non-preserved } \\
\text { Sterile 3\% hydrogen peroxide }\end{array}$ \\
\hline Ophtecs & Cleadew & Povidone-lodine \\
\hline \multirow[t]{3}{*}{ Allergan } & Hydrocare* & Thimerosal $0.002 \%$ \\
\hline & Complete* & Polyhexamethylene biguanide $0.0001 \%$ \\
\hline & ULTRACARE (Oxysept in the UK) & $\begin{array}{l}\text { Non-preserved } \\
\text { Sterile } 3 \% \text { hydrogen peroxide }\end{array}$ \\
\hline
\end{tabular}


Table I (Continued).

\begin{tabular}{|l|l|l|}
\hline Manufacturer & Solution Name & Preservative(s) \\
\hline Abbott Medical Optics & RevitaLens OcuTec & $\begin{array}{l}\text { Alexidine dihydrochloride } 0.00016 \% \\
\text { Polyquaternium- } 10.0003 \%\end{array}$ \\
\hline Menicon & MeniCare Soft & Polyhexamethylene biguanide $0.0001 \%$ \\
\hline Softcon & Septicon* & Thimerosal $0.004 \%$ (second step with $3 \%$ hydrogen peroxide) \\
\hline
\end{tabular}

Note: *No longer commercially available.

similar in size and mechanism to thimerosal. ${ }^{24}$ While chlorhexidine is technically a biguanide, ${ }^{34}$ it is an older formulation compared to the biguanides used in modern MPS (outlined in Modern High Molecular Weight Preservatives). In the 1970 s and early 1980s, many CL solutions contained thimerosal, chlorhexidine, or both. Studies demonstrated that chlorhexidine was generally safe $^{35,36}$ and did not cause delayed hypersensitivity. However, chlorhexidine may act as a direct irritant. ${ }^{37}$ Overall, chlorhexidine is minimally toxic due to its slow release from CLs, and it is therefore only found in low concentrations in the tear film. This is evidenced by a study in which CLs treated with chlorhexidine displayed slowly decreasing, but persistent, antibacterial activity with repeated transfer to fresh agar plates seeded with bacteria. $^{37}$

Consequently, chlorhexidine was the logical next contender for use in MPS considering its improved safety profile compared to thimerosal, as well as its effectiveness against microbes. Chlorhexidine has been proven effective against in vitro Acanthamoeba even at short exposure times. $^{21,22}$ Shih et al found in 1991 that chlorhexidine was effective against Fusarium and Aspergillus fungi, while PQ-1 and PHMB were not, according to FDA standards. $^{38}$ A later in vitro study clarified further that chlorhexidine was only effective (as defined by the Clinical and Laboratory Standards Institute M38-A document) against Fusarium and Aspergillus at concentrations above $0.006 \%$; however, it was commonly available for purchase in $0.003 \%$ concentration MPS. ${ }^{20}$

There have been conflicting reports regarding the efficacy of chlorhexidine against bacteria. A 1991 study showed chlorhexidine significantly reduced the in vitro bacterial load of $P$. aeruginosa, S. aureus, and S. marcescens within four hours at normal concentrations $(0.005 \%) .{ }^{38}$ However, a 1996 study reported that MPS preserved with $0.00025 \%$ chlorhexidine was not able to reduce the load of $S$. marcescens or $P$. aeruginosa, ${ }^{33}$ particularly when adhered to CLs. The conclusion drawn from these results is that $S$. marcescens and $P$. aeruginosa became resistant to chlorhexidine ${ }^{39}$ however, the differing concentrations may well explain the discrepancy between the two studies.

One study found that chlorhexidine had little effect on in vitro rabbit corneal epithelium in concentrations likely to be found in human tear film after three hours of CL wear, and that complications only arose at concentrations unlikely or impossible to achieve in in vivo environments with CLs. ${ }^{36}$ Chlorhexidine was not explored in as much depth, likely because newer and better high molecular weight preservatives were on the horizon. Like thimerosal, chlorhexidine is no longer used in modern CL MPS.

The combination of thimerosal and chlorhexidine in MPS causes more ocular surface adverse events than either preservative alone. A retrospective chart review of 161 patients using MPS containing chlorhexidine plus thimerosal found an incidence of solution intolerance (indicated by corneal staining) of $33 \%$ versus intolerance of only $5 \%$ to $8 \%$ in patients using MPS with thimerosal alone. ${ }^{30}$ Conversely, in a study of 38 patients with conjunctivitis and keratitis related to soft CL wear using MPS containing thimerosal plus chlorhexidine, 31 responded to a challenge with one drop of $0.004 \%$ thimerosal in one eye every two hours during waking hours for one day, while only 3 reacted to a skin patch test of $1 \%$ chlorhexidine. ${ }^{25}$ This suggests that thimerosal was the main irritating agent when both preservatives were combined, and chlorhexidine minimally contributed to adverse reactions. ${ }^{25}$ The literature conflicts on which preservative is more toxic to the ocular surface; however, neither is found in modern MPS.

Benzalkonium chloride (BAK) is a quaternary ammonium compound often used as a preservative in topical ocular medications due to its long shelf-life and effective antimicrobial properties. ${ }^{40}$ BAK is not used in CL MPS, however, because it is easily absorbed and released by CLs and has a well-documented history of cytotoxicity. ${ }^{41,42}$ 


\section{Modern High Molecular Weight Preservatives}

Modern preservatives are composed of larger, higher molecular weight molecules than thimerosal or chlorhexidine. The size of these preservatives makes them safer for the eye, as there is less risk of uptake and release from CLs, minimizing the risk to the ocular surface. ${ }^{43} \mathrm{PQ}-1$ and PHMB are now the most commonly used preservatives in commercially available MPS. In fact, PHMB-based MPS comprise $60 \%$ of the United States market and most of the Chinese market. ${ }^{20}$ Several different PQ-1 MPS formulations are on the market (Table 1). The frequency of PHMB and PQ-1 use has spurred a wealth of knowledge related to these products. The following section describes these common high molecular weight preservatives.

One of the most common high molecular weight preservatives are the biguanides. Biguanides invade microbial cells and interact with DNA in a way that disrupts DNA function and/or precipitation, causing cell death. ${ }^{44}$ Biguanides commonly found in modern CL MPS include polyhexamethylene biguanide (PHMB), polyaminopropyl biguanide, polyhexanide, and alexidine; sometimes grouped together and abbreviated as "PHMB" because they are all derived from the PHMB molecule. Hereafter, in this paper, all biguanides will be referred to as "PHMB" unless necessary to specify the particular molecule being discussed.

PHMB-based solutions have been shown to be effective in vitro against bacteria, specifically $P$. aeruginosa and S. aureus, ${ }^{14,39}$ according to both $\mathrm{FDA}^{38,45,46}$ and $\mathrm{ISO}^{46-48}$ standards. Other bacteria that are reported to be susceptible to PHMB solutions in vitro are $S$. pneumoniae ${ }^{45,47}$ and Streptococcus pyogenes. ${ }^{47}$ Some studies have more disagreement over other bacterial organisms' susceptibility to PHMB. One study claims PHMB effectively reduces the bacterial load of in vitro Escherichia coli and Staphylococcus epidermidis according to FDA guidelines, ${ }^{45}$ while another claims PHMB is ineffective against clinical isolates of these bacteria, ${ }^{14}$ though the authors of the latter study concede that when PHMB is combined with polyaminopropyl biguanide, they are effective. Four studies report PHMB is effective against S. marcescens, ${ }^{14,39,45,46}$ but one claims it is not effective. ${ }^{38}$ Two studies stipulate that PHMB is not effective against high-concentration planktonic bacterial accumulations or biofilms that may form on CLs. ${ }^{33,49}$ Another claims PHMB is effective against clinical isolates of these common causative bacteria when adhered to silicone hydrogel CLs only after full completion of manufacturer cleaning recommendations, and not after any of the intermediate steps (must be rubbed and rinsed, stored at minimum manufacturer recommended time, and rinsed again before application) ${ }^{50}$ Overall, the above studies indicate adequate efficacy of PHMB solutions against bacteria.

Unfortunately, PHMB MPS are not as effective against fungal, yeast, or amoebal species. The studies outlined above indicate that PHMB is effective against bacteria, but it is ineffective against Aspergillus ${ }^{20,38,45}$ or Candida species. ${ }^{38,45}$ Fusarium spp. are susceptible to PHMB only under specific conditions. PHMB was shown to only be effective against Fusarium and Aspergillus at concentration above $0.005 \%$, but PHMB MPS are available in concentrations as low as $0.001 \% .{ }^{20}$ Similarly, a study showed one MPS with $0.001 \%$ PHMB was effective against ISO and FDA standard fungi, but two others with the same concentration were not, ${ }^{46}$ demonstrating the importance of the other components of a MPS. Additionally, one study showed that MPS with combination PHMB and alexidine were initially effective against Fusarium on CLs, but it lost efficacy after soaking for six hours in standard CL cases due to uptake into the CLs. ${ }^{41}$ This is an important consideration for CL wearers who reuse or top off solution, as the solution will no longer be effective against Fusarium after the initial use.

Studies have confirmed that PHMB solutions are completely ineffective against Acanthamoeba (five MPS tested with some overlap of MPS formulas in the studies). ${ }^{10,21-23,47,51-54}$ While bacteria are much more common causative agents of MK, fungal or amoebal infections can quickly become sight-threatening and must also be considered.

In addition to sub-par efficacy against non-bacterial microbes, PHMB also has a rather high incidence of ocular toxicity. Many in vivo studies have reported higher grade corneal staining in patients using PHMB MPS compared to PQ-1 MPS when wearing both hydrogel ${ }^{55}$ and silicone hydrogel $\mathrm{CLs}^{55-60}$ (worse with silicone hydrogel CLs). This was confirmed with Choy et al's 2012 in vitro analysis with atomic spectroscopy with the authors reporting that PHMB caused irreversible damage to cultured corneal epithelial cells and that $30 \%$ of cells' membranes were destroyed after 15 minutes; however, the solution tested contained boric acid, which is known to cause damage to the corneal epithelium. Conversely, with a similar in vitro spectroscopy study, Imayasu et al reported in 2008 that 
PHMB is not disruptive to corneal epithelial tight junctions. $^{61}$

Some studies found differences among PHMBcontaining solutions. In one study, two PHMB solutions were not at all cytotoxic with in vitro assays of immortalized human corneal epithelial cells, while one was moderate by assessment of cell viability assay, cell membrane integrity, and surface appearance by light microscopy compared to control solutions. ${ }^{42}$ Another study showed two PHMB solutions were not at all cytotoxic at any concentration tested $(1.25 \%, 2.5 \%, 5 \%$, and $10 \%)$, while one PHMB solution was cytotoxic at medium to high concentrations ( $5 \%$ and $10 \%$ ), and two other PHMB solutions were highly cytotoxic at all concentrations. ${ }^{62}$ Related clinical studies generally describe differences in clinical signs and symptoms among patients using PHMB-based solutions. ${ }^{57,58}$ These studies imply that concentration and ancillary chemicals like chelating and wetting agents can significantly influence how MPS affect the ocular surface.

PQ-1 is a quaternary ammonium compound commonly used in CL care products. PQ-1 is bactericidal, as its mechanism of action involves denaturation of proteins in bacterial cell walls. ${ }^{63}$ However, PQ-1 is only weakly effective against Fusarium solani ${ }^{64}$ and Candida albicans, ${ }^{65}$ and it has no effect against other fungi or amoeba (outlined later in this section). Therefore, PQ-1 is found in combination with amidoamine or PHMB compounds in modern MPS. Myristamidopropyl dimethylamine (Aldox), an amidoamine, effectively inhibits fungi and amoebae via cationic disruption of cell membranes. ${ }^{50,63}$

Similar to PHMB, studies disagree on whether PQ-1 is effective against $S$. marcescens. Three studies found PQ-1 to be effective, ${ }^{45,46,66}$ while three failed to find a meaningful reduction in the bacterial load. ${ }^{14,38,39}$ Hume et al clarified further that PQ-1 plus Aldox is effective against ISO standard strains, but this combination is not effective against clinically isolated $S$. marcescens. This indicates that there may be increased resistance of S. marcescens since the standards were set by the ISO. ${ }^{15}$ The studies above examined MPS containing both PQ-1 and Aldox; however, Abjani et al also evaluated PQ-1 plus alexidine, and they found the same results against all studied organisms, regardless of whether PQ-1 was paired with Aldox or alexidine in MPS. ${ }^{47}$

For Fusarium spp., Rosenthal et al reported that PQ-1 plus Aldox was initially effective and maintained a low fungal load for seven days. This contrasted with PHMB, which quickly lost efficacy during the same time period.
As previously stated, the longevity of the antimicrobial activity is important to consider for any patient who may reuse MPS solution. ${ }^{41}$

The efficacy of PQ-1-containing MPS on Acanthamoeba is a topic of debate. Studies that assessed the efficacy of isolated PQ-1 alone found that it was not effective against Acanthamoeba. ${ }^{21-23,51,54}$ Studies vary on the effectiveness of PQ-1 MPS against Acanthamoeba when combined with an additional preservative. Studies assessing the effectiveness of PQ-1 plus Aldox against Acanthamoeba show the combination is no more effective than PQ-1 alone. Four studies reported no efficacy against clinical and/or tap water isolates of Acanthamoeba cysts or trophozoites (100\% survival of all strains), ${ }^{4,52-54}$ while two studies reported the combination reduced the load of both cysts and trophozoites of an ATCC strain, ${ }^{10,63}$ indicating there may be a difference in susceptibility to PQ-1 plus Aldox between clinical and standard isolates of Acanthamoeba. Abjani et al also assessed PQ-1 plus alexidine with regards to its effectiveness against Acanthamoeba and reported that, similar to their findings for PQ-1 plus Aldox, this combination of preservatives is ineffective against Acanthamoeba. ${ }^{47}$

Codling et al used atomic absorption spectrophotometry to examine in vitro microbial cells treated with PQ-1 or Aldox. They assessed for potassium leakage, an indication that cell membranes had been disrupted. The authors found that PQ-1 caused potassium leakage from P. aeruginosa, $S$. marcescens, $S$. aureus while Aldox caused leakage from C. albicans and A. fumigatus. This confirms that PQ-1 is effective against bacteria, while Aldox is effective against fungi. Neither PQ-1 nor Aldox caused potassium leakage from Acanthamoeba, indicating this combination of preservatives is not effective against Acanthamoeba. ${ }^{65}$ As for biofilms, $P$. aeruginosa and $S$. aureus biofilms adherent to CLs were shown to be susceptible to PQ-1, but the preservative was ineffective against $S$. marcescens biofilms. ${ }^{49}$

Studies disagree on the importance of strict adherence to MPS manufacturer recommendations when determining the efficacy of PQ-1. Correa et al (as previously outlined in this section) reported in 2018 that PQ-1 plus Aldox MPS (and PHMB and PQ-1 plus PHMB MPS) was effective against $S$. aureus, $S$. epidermidis, $S$. pneumoniae, P. aeruginosa, and Enterobacteriaceae, but this was only true after completion of the full manufacturer's cleaning recommendations, including both rinsing and rubbing steps. ${ }^{50}$ Prior to this, Rosenthal et al's work in 2000 reported that PQ-1 plus Aldox MPS was effective against 
bacteria, fungi, and amoeba after only a 6-hour soak, indicating the rubbing step may be unnecessary. ${ }^{64}$

As for safety of PQ-1, a Phase I clinical trial showed safety at a concentration of $0.005 \%$, which is five times the commercially available concentration. Adverse events were low in the trial of 50 subjects, with only two instances of minimal corneal staining and no reports of corneal edema, conjunctival injection, or iritis. Additionally, only seven patients experienced mild irritation (minimal punctate staining or mild limbal injection) at higher concentrations, and none of these seven patients had any reaction when challenged with switching to daily use of a PQ-1 solution with the commercially available concentration $(0.001 \%)$, following manufacturer care instructions. ${ }^{18}$ Interestingly, one study reported a small number $(n=13)$ of patients using PQ-1 MPS presenting with dendritiform keratitis, that were determined to be not infectious and that resolved after discontinuation of PQ-1. This indicates that PQ-1-induced keratitis needs to be considered in the differential diagnosis of patients presenting with dendritiform lesions. ${ }^{67}$

Studies that evaluated both PHMB and PQ-1 MPS separately found these preservatives are equally effective against an array of microbes. Some studies agree that both preservatives were effective against $P$. aeruginosa, S. aureus, ${ }^{38,45-48}$ S. epidermidis, ${ }^{14,39,45}$ E. coli, and S. pneumoniae, ${ }^{4,47}$ while they were not effective against C. albicans or Aspergillus spp. ${ }^{38,45}$ One study found that PQ-1 plus Aldox was effective against $C$. albicans and Aspergillus spp, ${ }^{66}$ but no further corroborating information could be detected during this review.

Studies that directly compared the efficacy of PHMB and PQ-1 claim that PHMB is more effective against S. marcescens, S. aureus, and $P$. aeruginosa, ${ }^{46,48}$ but that PQ-1 plus Aldox MPS are more effective against in vitro Acanthamoeba than PHMB MPS. ${ }^{10}$ This does not necessarily translate to clinical efficacy of PQ-1 plus Aldox MPS, particularly because there are no standards for assessing effectiveness against Acanthamoeba.

Interestingly, one study found that PQ-1 plus alexidine and PQ-1 plus PHMB solutions were more effective against gram-negative bacteria that cause corneal infiltrative events than PQ-1 plus Aldox MPS, indicating that biguanides are likely more effective against these organisms. $^{68}$ Another study concurred, reporting PQ-1 plus Aldox was associated with more corneal infiltrative events compared to PHMB with daily wear CLs in a university population. ${ }^{69}$ Comparing the overall efficacy of PHMB versus PQ-1 MPS, the studies above generally indicate that PHMB and PQ-1 plus Aldox (or in a few cases PQ-1 plus alexidine) are equally effective against bacteria and equally ineffective against fungi; however, PQ-1 plus Aldox is superior to PHMB MPS in efficacy against Acanthamoeba.

Since PHMB and PQ-1 solutions have similar efficacy against most microbes, a direct comparison of their effects on the ocular surface could help determine which MPS are best to recommend to patients. While the findings of corneal staining seem unfavorable with PHMB, a study directly comparing corneal staining for PHMB versus PQ-1 MPS showed that PQ-1 had a higher incidence of central staining, and the solutions were equal for overall corneal staining, indicating PHMB may not be any worse than PQ-1 for staining. ${ }^{70}$ In support of this, one study found that PQ-1 plus Aldox was more cytotoxic by spectroscopy evaluation of human corneal epithelial cells than three PHMB-containing MPS. $^{42}$ Another study confirmed that PHMB is potentially worse than PQ-1 for corneal staining, reporting that staining was worse when PQ-1 was paired with a PHMB (alexidine) compared to a non-PHMB (Aldox) preservative. This occurred to a greater degree with silicone hydrogel CLs than with hydrogel CLs, although the authors concede all corneal staining was less than grade 1 on the Efron scale, which is generally considered clinically acceptable. ${ }^{71}$ In vitro studies to confirm PHMB and PQ-1 cytotoxicity to corneal epithelial cells also had conflicting results. As previously stated, Wright and Mowrey-McKee reported PQ-1 plus Aldox MPS was more cytotoxic by spectroscopy evaluation of human corneal epithelial cells than three PHMB MPS. ${ }^{42}$ Similarly, Choy et al's 2012 paper reported that PHMB caused irreversible damage to cultured corneal epithelial cells after 15 minutes, but PQ-1 solutions were still damaging at even shorter exposure times (10 minutes). The authors concluded that PHMB was less toxic than PQ-1 because it required a longer exposure time to disrupt the cell membrane and metabolic activity of the cell. $^{60}$ Imayasu et al agreed that both PHMB (alexidine) MPS and PQ-1 MPS were cytotoxic through disruption of epithelial cell tight junctions, but also postulated that the boric acid in these MPS could be the cause of the disruption, not the PHMB or PQ-1. ${ }^{61}$

While reports on corneal staining seem initially concerning with regards to risk for infection, the clinical implications of solution-induced staining are debatable. 
The corneal epithelium is vital to the barrier function of the cornea, preventing microbial invasion and infection. However, it is unclear whether there is epithelial disruption from solution-induced staining and, if present, whether it is transient or concerning. Studies have shown that solution-induced staining is significantly worse during the first two to four hours of CL wear. ${ }^{55-57}$ Additionally, studies vary on how this staining affects patient comfort. Jones reported there was no difference in subjective symptoms other than slightly more stinging on application with PHMB MPS than PQ-1 MPS, ${ }^{56}$ while two studies reported that as staining increased, comfort decreased. ${ }^{57,59}$

In addition to corneal findings, there are clinical reports of negative effects of PHMB on the conjunctiva (staining, papillae, hyperemia) when compared to $\mathrm{PQ}-1^{58,59}$ and $\mathrm{H}_{2} \mathrm{O}_{2} \cdot{ }^{72,73}$ In support of the clinical findings, Dutot et al reported in 2008 that three PHMB MPS caused oxidative stress and apoptosis in human conjunctival cells. ${ }^{74}$ However, conjunctival apoptosis was also found with one PQ-1 plus Aldox solution, ${ }^{74}$ so the negative effects may be similar between PHMB and PQ-1 MPS.

When comparing the additional preservative in PQ-1 solutions, Aldox causes more stinging, redness, and conjunctival staining than alexidine upon CL application, but there is no difference in corneal staining seen between the two. $^{75}$ There is also one case report published showing PHMB MPS caused contact dermatitis on the skin of the hand, ${ }^{34}$ but no reports have been published on dermatologic adverse events related to PQ-1 use.

One MPS combines both PHMB and PQ-1 as preservatives. It would be reasonable to assume that this solution is more effective than MPS with stand-alone preservatives, but it is more likely to cause complications. The literature confirms that the combination may be more effective. A study isolating standard strains of $P$. aeruginosa and S. aureus found that a PQ-1 plus PHMB MPS was more effective at a faster disinfecting time (four hours) and higher overall $\log$ reduction in bacteria $(5 \log )$ than standalone PQ-1 (six hours, 5log) or PHMB (failed to meet requirement of $6 \mathrm{log}$ reduction at manufacturer recommended six hour disinfection time). ${ }^{76}$ Fortunately, the research refutes the hypothesis that combining PQ-1 and PHMB in MPS would be less safe than MPS with one preservative. An extensive meta-analysis of six clinical trials $(3,134$ eyes, 72,904 daily exposures) showed the combination of PQ-1 and PHMB had a very small percentage of ocular surface adverse events graded $>2+$ on a 0 to 4 ordinal system $(0=$ none; $1=$ trace; $2=$ mild; $3=$ moderate; $4=$ severe) where findings graded $<2+$ were considered as not requiring treatment. ${ }^{77}$ One study did find that PQ-1 plus PHMB MPS was inferior to $\mathrm{H}_{2} \mathrm{O}_{2}$ for corneal staining with one hydrogel and one silicone hydrogel CL, but not with another silicone hydrogel CL, and that the combination was equal in bulbar and limbal redness and in subjective comfort to $\mathrm{H}_{2} \mathrm{O}_{2}{ }^{71}$ These findings were also found with PQ-1 plus alexidine and PQ-1 plus Aldox, indicating that PQ-1 plus PHMB is not inferior to the other combinations. It seems the combination of the two does not cause any additional ocular surface adverse events when compared to either preservative alone. It is also reasonable to assume that the combination of PHMB plus PQ-1 in MPS may not be effective against Acanthamoeba, considering the studies above indicate that neither preservative alone is effective against amoebas. Indeed, one study confirmed this solution is not effective against Acanthamoeba. ${ }^{78}$

In summary, PQ-1 and PHMB are generally equal in efficacy and safety. They are equally effective against bacteria, minimally effective against fungi, and equally ineffective against Acanthamoeba. PQ-1 and PHMB also have similar safety profiles. Both have been shown to cause conjunctival disruption and corneal staining. PHMB may have a slightly higher frequency of corneal staining, particularly with silicone hydrogel CLs, although the studies are conflicting as to whether this staining is clinically relevant and whether it is truly worse with PHMB than with PQ-1. Ultimately, all CL patients should be monitored for ocular adverse events.

While it is important to consider the safety and efficacy of solutions associated with CL packaging, particularly with the popularity of daily disposable CLs and repeated daily exposure to their blister pack solutions (BPS), BPS do not contain preservatives. Additionally, most commercially available soft CLs are sealed in a sterile manufacturing environment, and the CL coming out of the packing solution is directly applied to the eye with minimal potential exposure to microbes. Considering preservatives are not involved, and the risk for MK upon removal from BPS is low, it is not of critical importance to consider BPS in this context.

\section{Non-Preserved CL Care Solutions Hydrogen Peroxide Solutions}

Hydrogen peroxide $\left(\mathrm{H}_{2} \mathrm{O}_{2}\right)$ is not classified as a preservative, but it is commonly used to disinfect CLs due to its excellent antimicrobial efficacy. $\mathrm{H}_{2} \mathrm{O}_{2}$ is a broad- 
spectrum disinfectant that works by oxidizing all types of microbes, ${ }^{63}$ destroying cell membranes by changing the bonds between lipids and proteins, which alters the membrane's permeability. ${ }^{63,79}$ After CLs are disinfected with $\mathrm{H}_{2} \mathrm{O}_{2}$, the solution is neutralized into phosphate-buffered saline with either a platinum disc or catalase tablet for a minimum of six hours. ${ }^{21,80,81} \mathrm{H}_{2} \mathrm{O}_{2}$-treated lenses can be applied directly to the eye with minimal risk of ocular surface damage or allergic reaction after neutralization, as the neutralized saline is preservative-free. Nevertheless, the risk is not zero due to buffers present in the neutralized solution, and some practitioners advocate for a rinse with saline prior to CL application. ${ }^{79}$

$\mathrm{H}_{2} \mathrm{O}_{2}$ cleaning systems are often used as the comparative standard in studies evaluating the safety and efficacy of preserved CL solutions because they are safe and have been proven broadly effective. One review of $\mathrm{H}_{2} \mathrm{O}_{2}$ systems states "data indicates that in comparison with MPS, onestep $\mathrm{H}_{2} \mathrm{O}_{2}$ systems tend to promote more favorable compliance, efficacy, comfort, and ocular surface outcomes" and even goes as far as to recommend that eye care practitioners consider making one-step $\mathrm{H}_{2} \mathrm{O}_{2}$ systems their firstline CL care recommendation for most wearers of reusable CLs. ${ }^{79}$ However, some authors note $\mathrm{H}_{2} \mathrm{O}_{2}$ systems are less convenient to use since they require neutralization before contact with the eye, and they may need to be used in conjunction with additional cleaning products. ${ }^{82,83}$ Further head-to-head studies of $\mathrm{H}_{2} \mathrm{O}_{2}$ systems and MPS could elucidate the best compromise between convenience, safety profile, and antimicrobial activity.

There is extensive evidence to indicate that $\mathrm{H}_{2} \mathrm{O}_{2}$ systems, which do not require use of preservatives, are quite effective against a wide array of organisms compared to MPS. Early studies in the late 1990s comparing the efficacy of $\mathrm{H}_{2} \mathrm{O}_{2}$ systems to chemical systems had varying results depending on the organisms tested. Two studies examined the effectiveness of solutions against Staphylococcus spp., P. aeruginosa, S. marcescens, Candida spp., and Aspergillus spp. They found that MPS preserved with PQ-1 plus Aldox ${ }^{66}$ and PHMB $^{14}$ were similarly as effective as $\mathrm{H}_{2} \mathrm{O}_{2}$ against fungal species, ${ }^{14,66}$ were less effective but still within accepted guidelines against bacterial species, ${ }^{14,66}$ and had the important advantage of long-term antimicrobial activity that is not possible with $\mathrm{H}_{2} \mathrm{O}_{2}$ systems due to neutralization. ${ }^{66}$ In contrast to the similar efficacy found with all MPS against single colony bacteria harvested on agar plates, $\mathrm{H}_{2} \mathrm{O}_{2}$ was the only solution found to be effective against all strains of bacteria that commonly cause corneal ulcers when they form biofilms on silicone hydrogel CLs. ${ }^{49}$

Similar studies have examined the microbicidal activity of $\mathrm{H}_{2} \mathrm{O}_{2}$ specifically against Acanthamoeba. Studies have specifically harvested Acanthamoeba strains and challenged them in vitro. In these studies Acanthamoeba were placed in containers provided by the manufacturers, in well-plates, or in a disinfection solution suspension for the manufacturerrecommended disinfecting times with commercially available doses of $\mathrm{H}_{2} \mathrm{O}_{2}$, PHMB MPS, or PQ-1 MPS in all studies, ${ }^{21,23,51}$ in addition to BAK and chlorhexidinethimerosal in two studies. ${ }^{23}$ The studies concluded that $\mathrm{H}_{2} \mathrm{O}_{2}$ was most effective at the shortest storage times against Acanthamoeba, while PQ-1 and PHMB were determined to be completely ineffective..$^{21,23,51}$ BAK and chlorhexidine-thimerosal were sufficient at killing both trophozoites and cysts depending on exposure time ${ }^{21,23}$ but, as previously noted, these preservatives are no longer used in commercially available MPS due to toxicity.

So long as full neutralization is achieved, it is rare for $\mathrm{H}_{2} \mathrm{O}_{2}$ systems to cause ocular surface compromise. It is well-documented that preserved MPS can cause ocular irritation, as outlined previously. Two studies compared $\mathrm{H}_{2} \mathrm{O}_{2}$ systems specifically to PHMB MPS regarding their effect on the eyelids, and both found that papillae and patient symptoms were improved after using $\mathrm{H}_{2} \mathrm{O}_{2}$ for 90 days. $^{72,73}$ It was previously outlined in Modern High Molecular Weight Preservatives how MPS negatively affects the cornea, and there was no evidence that $\mathrm{H}_{2} \mathrm{O}_{2}$ systems cause corneal adverse events after proper neutralization. However, one study did find that there was no difference in patient-reported average comfortable wear time or in observed corneal staining between CLs treated with a $\mathrm{H}_{2} \mathrm{O}_{2}$ system versus MPS with PQ-1 plus Aldox, PQ-1 plus PHMB, or PQ-1 plus alexidine, implying that MPS with modern preservatives create a comfortable and non-compromised ocular surface much like $\mathrm{H}_{2} \mathrm{O}_{2}$ systems. ${ }^{71}$ Overall, the studies confirm that $\mathrm{H}_{2} \mathrm{O}_{2}$ systems generally exhibit at least equal, and sometimes superior, antimicrobial efficacy along with an impressive safety profile compared to MPS containing preservatives. However, clinicians must consider the convenience and long-term antimicrobial effects of preserved MPS that $\mathrm{H}_{2} \mathrm{O}_{2}$ systems do not achieve.

\section{Povidone-lodine}

Povidone-Iodine (P-I) has long been used as a general disinfecting agent during surgery, including on the ocular 
surface before intraocular surgery, due to its broadspectrum antimicrobial capabilities but low cytotoxicity to human tissues. ${ }^{84}$ The mechanism of action of P-I is penetration of microbial cell walls and attack of proteins and DNA, causing apoptosis. ${ }^{85}$ While not yet commercially available in the United States, P-I solutions are used as CL disinfectants in Japan, Europe, Hong Kong, and New Zealand. ${ }^{85}$ The only currently commercially available P-I system includes a solution of boric acid buffer and $\mathrm{H}_{2} \mathrm{O}_{2}$, with a tablet that dissolves when added to the solution. The tablet is composed of an outer layer that contains P-I for disinfection, and an inner layer that includes a proteolytic enzyme for neutralization of the $\mathrm{H}_{2} \mathrm{O}_{2}$. ${ }^{85}$

A study evaluating both antimicrobial activity and potential cytotoxicity of P-I used as a CL disinfecting solution compared P-I to $\mathrm{H}_{2} \mathrm{O}_{2}$, PHMB MPS, and a BAK solution. This study found P-I was both the most effective disinfectant against the tested bacteria and fungus and the least cytotoxic of the four solutions, ${ }^{83}$ making it a promising recent innovation in the $\mathrm{CL}$ disinfection market. Another in vitro study corroborated that P-I is effective against S. aureus and P. aeruginosa. ${ }^{48}$ A recent study has also confirmed that P-I is effective against clinical isolates of bacterial species ( $P$. aeruginosa from a patient with $\mathrm{MK}$; and S. marcescens and Achromobacter xylosoxidans from CL cases), even in the presence of organic soils, and reports no adverse events due to P-I. ${ }^{13}$ Furthermore, a study comparing a P-I MPS to a PQ-1 plus PHMB MPS found they were both effective against in vitro planktonic bacteria (standard strain S. marcescens; and clinically isolated P. aeruginosa, Delftia acidovorans, Stenotrophomonas maltophilia, S. aureus, and S. epidermidis), but only P-I was effective against biofilms isolated from CL cases. ${ }^{85}$ Studies have also proven P-I is effective against in vitro clinically isolated $^{86}$ and known pathogenic ${ }^{87,88}$ strains of Acanthamoeba cysts and trophozoites. From these studies, P-I can achieve and even greatly exceed safety standards for CL MPS and is safe for ocular use in CL disinfection.

Considering its excellent efficacy and safety profile, P-I solutions may be forthcoming in North America. However, it must be considered that the only currently available P-I solution evaluated in these studies also contains $\mathrm{H}_{2} \mathrm{O}_{2}$, which is known to be effective on its own. Therefore, it is impossible to determine from these studies how effective P-I is as a stand-alone preservative. Further studies are needed to determine the validity of more widespread use of this preservative.

\section{Conclusions}

CL solutions containing $\mathrm{H}_{2} \mathrm{O}_{2}$, PHMB, P-I, or PQ-1 are all potentially viable care system options for soft CLs. It is worth considering that PHMB is a common cause of corneal staining, particularly in patients using silicone hydrogel CLs. While the clinical implications of solution-induced corneal staining may be up for debate since this type of staining is usually transient and may not increase the risk for infection, there is no harm in switching patients to a solution with a different preservative should this staining occur. While PQ-1 solutions may cause fewer ocular surface adverse events than PHMB MPS, events are still common and need to be monitored. If corneal staining persists with both PHMB and PQ-1 preserved MPS, it can be assumed that ancillary components are causing the staining and switching to an $\mathrm{H}_{2} \mathrm{O}_{2}$ solution should be considered as it is neutralized into phosphate-buffered saline with minimal additives. For patients who can properly and conveniently follow manufacturers' guidelines for use of $\mathrm{H}_{2} \mathrm{O}_{2}$ solutions, this may be the best option for both safety and efficacy, as $\mathrm{H}_{2} \mathrm{O}_{2}$ is effective against all microbes and poses almost no threat to the ocular surface when neutralized. While Acanthamoeba infections are rare, they can quickly become sight-threatening, raising some concern over the fact that neither PHMB nor PQ-1 based solutions are effective against this microbe. Patients who expose their CLs to water are at a significantly increased risk for Acanthamoeba infection, so $\mathrm{H}_{2} \mathrm{O}_{2}$ or P-I should be recommended as they are the only solutions that are definitively clinically effective against Acanthamoeba.

Recall that MPS studies may vary depending on study design, study duration, and additional components in the solution (other than the preservatives), so outcomes should be monitored clinically. While various studies have assessed multiple MPS with different preservatives, or MPS containing more than one preservative, many have yielded conflicting results, and none make definitive recommendations for the use of one preservative over another. Further studies examining specific differences between MPS with different preservatives would be helpful for the field to make clinical recommendations. Eye care practitioners should tailor their CL solution recommendations based on a patient's specific needs, with consideration for their occupational and recreational environments, ease of handling, ability to properly follow manufacturer instructions for disinfection, the type of $\mathrm{CL}$ they are using, and the clinical presentation of their ocular 
surface. Ultimately, the market lacks a perfect CL care system, but continued innovations keep the field progressing towards this goal.

\section{Acknowledgments}

No funding was specifically provided for the completion of this work. The authors thank the journal for inviting them to complete this literature review.

\section{Funding}

There is no funding to report.

\section{Disclosure}

Dr. Lindsay A. Sicks reports personal fees and Honoraria (Speaker's Alliance) from Alcon Laboratories outside the submitted work. Dr. Andrew D. Pucker has received financial research support from Alcon Research, LLC, Art Optical, Bausch \& Lomb, Euclid Systems, Contamac, National Eye Institute, and Paragon Vision Sciences, and consulted for Alcon Research, LLC, CooperVision, EpiTech, EyeGate Pharmaceuticals, Inc, Kala Pharmaceuticals, Nevakar Inc, and Optikal Care Inc over the past three years. The authors report no other conflicts of interest in this work.

\section{References}

1. Whitcher JP, Srinivasan M, Upadhyay MP. Corneal blindness: a global perspective. Bull World Health Organ. 2001;79(3):214-221.

2. Wilhelmus KR. Review of clinical experience with microbial keratitis associated with contact lenses. CLAO J. 1987;13(4):211-214.

3. Agi J, Rocchetti TT, Yu MCZ, et al. Three decades of contact lensassociated microbial keratitis in a referral Hospital in São Paulo, Brazil. Arq Bras Oftalmol. 2020;82:7.

4. Lam DSC, Houang E, Fan DSP, et al. Incidence and risk factors for microbial keratitis in Hong Kong: comparison with Europe and North America. Eye. 2002;16(5):608-618. doi:10.1038/sj.eye.6700151

5. Stapleton F, Carnt N. Contact lens-related microbial keratitis: how have epidemiology and genetics helped us with pathogenesis and prophylaxis. Eye. 2012;26(2):185-193. doi:10.1038/eye.2011.288

6. Stapleton F, Naduvilath T, Keay L, et al. Risk factors and causative organisms in microbial keratitis in daily disposable contact lens wear. PLoS One. 2017;12(8):e0181343. doi:10.1371/journal.pone.0181343

7. Morgan P, Woods CA, Tranoudis IG, et al. International Contact Lens Prescribing in 2019. Contact Lens Spectrum; 2020. Avialable from: https://www.clspectrum.com/issues/2020/january-2020/internationalcontact-lens-prescribing-in-2019. Accessed July 30, 2021.

8. International Organization for Standardization. Ophthalmic opticsContact lens care products-Microbiological requirements and test methods for products and regimens for hygienic management of contact lenses; 2001. Available from: https://www.iso.org/standard/25382. html. Accessed July 30, 2021.

9. Rosenthal RA, Sutton SVW, Schlech BA. Review of standard for evaluating the effectiveness of contact lens disinfectants. PDA J Pharm Sci Technol. 2002;56(1):37-50.
10. Buck SL, Rosenthal RA, Schlech BA. Amoebicidal activity of multipurpose contact lens solutions. Eye Contact Lens. 2005;31(2):62-66. doi:10.1097/01.ICL.0000146322.63546.50

11. Borazjani RN, Kilvington S. Effect of a multipurpose contact lens solution on the survival and binding of Acanthamoeba species on contact lenses examined with a no-rub regimen. Eye Contact Lens. 2005;31(1):39-45. doi:10.1097/01.ICL.0000146303.00326.69

12. Gabriel MM, McAnally C, Bartell J. Antimicrobial efficacy of multipurpose disinfecting solutions in the presence of contact lenses and lens cases. Eye Contact Lens. 2018;44(2):125-131. doi:10.1097/ ICL.0000000000000308

13. Yamasaki K, Mizuno Y, Kitamura Y, Willcox M. The antimicrobial activity of multipurpose disinfecting solutions in the presence of different organic soils. Eye Contact Lens. 2020;46(4):201-207. doi:10.1097/ICL.0000000000000694

14. Cano-Parra J, Bueno-Gimeno I, Lainez B, Córdoba J, Montés-Micó R. Antibacterial and antifungal effects of soft contact lens disinfection solutions. Cont Lens Anterior Eye. 1999;22 (3):83-86. doi:10.1016/S1367-0484(99)80043-5

15. Hume EBH, Zhu H, Cole N, Huynh C, Lam S, Willcox MDP. Efficacy of contact lens multipurpose solutions against serratia marcescens. Optom Vis Sci. 2007;84(4):316-320. doi:10.1097/ OPX.0b013e3180465543

16. Hume EBH, Flanagan J, Masoudi S, Zhu H, Cole N, Willcox MDP. Soft contact lens disinfection solution efficacy: clinical Fusarium isolates vs. ATCC 36031 Optom Vis Sci. 2009;86(5):415-419. doi:10.1097/OPX.0b013e31819fa239

17. Food and Drug Administration. Contact lens care products - Premarket Notification 510(k) guidance; 1997. Available from: https://www.fda. gov/media/72725/download. Accessed July 30, 2021.

18. Morgan JF, Perry DL, Stein JM, Randeri KJ. The margin of safety of polyquaternium-1 preserved lens care solutions: a phase I clinical study. CLAO J. 1988;14(2):76-80.

19. Elferink JG. Thimerosal: a versatile sulfhydryl reagent, calcium mobilizer, and cell function-modulating agent. Gen Pharmacol. 1999;33(1):1-6. doi:10.1016/S0306-3623(98)00258-4

20. Xu Y, He Y, Zhou L, et al. Effects of contact lens solution disinfectants against filamentous fungi. Optom Vis Sci. 2014;91 (12):1440-1445. doi:10.1097/OPX.0000000000000407

21. Silvany RE, Dougherty JM, McCulley JP, Wood TS, Bowman RW, Moore MB. The effect of currently available contact lens disinfection systems on Acanthamoeba castellanii and Acanthamoeba polyphaga. Ophthalmology. 1990;97(3):286-290. doi:10.1016/S0161-6420(90) 32590-3

22. Silvany RE, Dougherty JM, McCulley JP. Effect of contact lens preservatives on Acanthamoeba. Ophthalmology. 1991;98 (6):854-857. doi:10.1016/S0161-6420(91)32210-3

23. Zanetti S, Fiori PL, Pinna A, Usai S, Carta F, Fadda G. Susceptibility of Acanthamoeba castellanii to contact lens disinfecting solutions. Antimicrob Agents Chemother. 1995;39(7):1596-1598. doi:10.1128/ AAC.39.7.1596

24. Jones L, Powell CH. Uptake and release phenomena in contact lens care by silicone hydrogel lenses. Eye Contact Lens. 2013;39 (1):29-36. doi:10.1097/ICL.0b013e31827d4f25

25. Rietschel RL, Wilson LA. Ocular inflammation in patients using soft contact lenses. Arch Dermatol. 1982;118(3):147-149. doi:10.1001/ archderm.1982.01650150009010

26. Jenkins C, Tuft S, Liu C, Buckley R. Limbal transplantation in the management of chronic contact-lens-associated epitheliopathy. Eye. 1993;7(Pt 5):629-633. doi:10.1038/eye.1993.145

27. Zeigen SR, Jacobs IH, Weinberger GI. Delayed hypersensitivity to thimerosal in contact lens solutions. J Med Soc $N$ J. 1981;78 (5):362-364.

28. Mondino BJ, Salamon SM, Zaidman GW. Allergic and toxic reactions of soft contact lens wearers. Surv Ophthalmol. 1982;26 (6):337-344. doi:10.1016/0039-6257(82)90126-6 
29. Sertoli A, Di Fonzo E, Spallanzani P, Panconesi E. Allergic contact dermatitis from thimerosol in a soft contact lens wearer. Contact Dermatitis. 1980;6(4):292-293. doi:10.1111/j.1600-0536.1980.tb04941.x

30. Coward BD, Neumann R, Callender M. Solution intolerance among users of four chemical soft lens care regimens. Am J Optom Physiol Opt. 1984;61(8):523-527. doi:10.1097/00006324-198408000-00006

31. Tripathi BJ, Tripathi RC, Kolli SP. Cytotoxicity of ophthalmic preservatives on human corneal epithelium. Lens Eye Toxic Res. 1992;9 (3-4):361-375.

32. Nguyen DQ, Srinivasan S, Hiscott $P$, Kaye SB. Thimerosal-induced limbal stem cell failure: report of a case and review of the literature. Eye Contact Lens. 2007;33(4):196-198. doi:10.1097/01.icl.000 0247636.10720 .19

33. Lehtonen OJ, Vaahtoranta-Lehtonen H, Leivo P, Mäki M. Synergistic effect of ethyl-6-O-decanoyl glucoside and chlorhexidine in contact lens disinfection. APMIS. 1996;104(7-8):603-606. doi:10.1016/ S0161-6420(01)00723-0

34. M-a P-N, González-Muñoz P, Pérez-Mesonero R, et al. Allergic contact dermatitis caused by poly(hexamethylene) biguanide hydrochloride in contact lens care solutions. Contact Dermatitis. 2017;76 (6):373-376. doi:10.1111/cod.12730

35. Gasset AR, Ishii Y. Cytotoxicity of chlorhexidine. Can J Ophthalmol. 1975;10(1):98-100.

36. Green K, Livingston V, Bowman K, Hull DS. Chlorhexidine effects on corneal epithelium and endothelium. Arch Ophthalmol. 1980;98 (7):1273-1278. doi:10.1001/archopht.1980.01020040125020

37. Refojo MF. Reversible binding of chlorhexidine gluconate to hydrogel contact lenses. Eye Contact Lens. 1976;2(1):47.

38. Shih KL, Raad MK, Hu JC, et al. Disinfecting activities of non-peroxide soft contact lens cold disinfection solutions. CLAO J. 1991;17(3):165-168.

39. Parment PA, Colucci B, Nyström B. The efficacy of soft contact lens disinfection solutions against Serratia marcescens and Pseudomonas aeruginosa. Acta Ophthalmol Scand. 1996;74(3):235-237. doi:10. 1111/j.1600-0420.1996.tb00083.x

40. Dracopoulos A, Dixon DG, Jones LW, Sivak JG, Bantseev V. In vitro assessment of medical device toxicity: interactions of benzalkonium chloride with silicone-containing and p-hema-containing hydrogel contact lens materials. Eye Contact Lens. 2007;33(1):26-37. doi:10.1097/01.icl.0000229775.17844.3f

41. Rosenthal RA, Dassanayake NL, Schlitzer RL, Schlech BA, Meadows DL, Stone RP. Biocide uptake in contact lenses and loss of fungicidal activity during storage of contact lenses. Eye Contact Lens. 2006;32(6):262-266. doi:10.1097/ICL.0b013e31802b413f

42. Wright A, Mowrey-McKee M. Comparative cytotoxicity potential of soft contact lens care products. Cutan Ocul Toxicol. 2005;24 (1):53-64. doi:10.1081/CUS-200046191

43. Green JA, Phillips KS, Hitchins VM, et al. Material properties that predict preservative uptake for silicone hydrogel contact lenses. Eye Contact Lens. 2012;38(6):350-357. doi:10.1097/ICL.0b013e318272c470

44. Allen MJ, White GF, Morby AP. The response of Escherichia coli to exposure to the biocide polyhexamethylene biguanide. Microbiology. 2006;152(Pt 4):989-1000. doi:10.1099/mic.0.28643-0

45. Lowe R, Vallas V, Brennan NA. Comparative efficacy of contact lens disinfection solutions. CLAO J. 1992;18(1):34-40.

46. Miller MJ, Callahan DE, McGrath D, Manchester R, Norton SE. Disinfection efficacy of contact lens care solutions against ocular pathogens. CLAO J. 2001;27(1):16-22.

47. Abjani F, Khan NA, Jung SY, Siddiqui R. Status of the effectiveness of contact lens disinfectants in Malaysia against keratitis-causing pathogens. Exp Parasitol. 2017;183:187-193. doi:10.1016/j.exppara. 2017.09.007

48. Manuj K, Gunderson C, Troupe J, Huber ME. Efficacy of contact lens disinfecting solutions against Staphylococcus aureus and Pseudomonas aeruginosa. Eye Contact Lens. 2006;32(4):216-218. doi:10.1097/01.icl.0000194922.53171.39
49. Szczotka-Flynn LB, Imamura Y, Chandra J, et al. Increased resistance of contact lens-related bacterial biofilms to antimicrobial activity of soft contact lens care solutions. Cornea. 2009;28(8):918-926. doi:10.1097/ICO.0b013e3181a81835

50. Correa PC, Lui ACF, Silva CB, et al. Study of the effectiveness of multipurpose solutions on the bacterial disinfection of silicone hydrogel contact lenses in vitro. Eye Contact Lens. 2018;44(Suppl 2):S24S28. doi:10.1097/ICL.0000000000000428

51. Stapleton F, Harmis N, Deshpande R, Tran D. Preliminary studies on the amoebicidal efficacy of contact lens disinfection systems. Aust $N$ Z J Ophthalmol. 1998;26(Suppl 1):S44-S46. doi:10.1111/j.14429071.1998.tb01369.x

52. Shoff ME, Joslin CE, Tu EY, Kubatko L, Fuerst PA. Efficacy of contact lens systems against recent clinical and tap water Acanthamoeba isolates. Cornea. 2008;27(6):713-719. doi:10.1097/ 01.ico.0000611416.57783.a7

53. Boost MV, Shi G-S, Lai S, Cho P. Amoebicidal effects of contact lens disinfecting solutions. Optom Vis Sci. 2012;89(1):44-51. doi:10.1097/OPX.0b013e31823ac85e

54. Johnston SP, Sriram R, Qvarnstrom Y, et al. Resistance of Acanthamoeba cysts to disinfection in multiple contact lens solutions. J Clin Microbiol. 2009;47(7):2040-2045. doi:10.1128/ JCM.00575-09

55. Garofalo RJ, Dassanayake N, Carey C, Stein J, Stone R, David R. Corneal staining and subjective symptoms with multipurpose solutions as a function of time. Eye Contact Lens. 2005;31(4):166-174. doi:10.1097/01.ICL.0000152489.99455.DB

56. Jones L, MacDougall N, Sorbara LG. Asymptomatic corneal staining associated with the use of balafilcon silicone-hydrogel contact lenses disinfected with a polyaminopropyl biguanide-preserved care regimen. Optom Vis Sci. 2002;79(12):753-761. doi:10.1097/000 06324-200212000-00007

57. Andrasko G, Ryen K. Corneal staining and comfort observed with traditional and silicone hydrogel lenses and multipurpose solution combinations. Optometry. 2008;79(8):444-454. doi:10.1016/j.optm. 2008.04.097

58. Young G, Keir N, Hunt C, Woods CA. Clinical evaluation of long-term users of two contact lens care preservative systems. Eye Contact Lens. 2009;35(2):50-58. doi:10.1097/ICL.0b013e31819630d3

59. Lipener C. Contact Lens Advisory in Scientific Studies (CLASS) group. A randomized clinical comparison of OPTI-FREE EXPRESS and $\mathrm{ReNu}$ MultiPLUS multipurpose lens care solutions. Adv Ther. 2009;26(4):435-446.

60. Choy CKM, Cho P, Boost MV. Cytotoxicity and effects on metabolism of contact lens care solutions on human corneal epithelium cells. Clin Exp Optom. 2012;95(2):198-206. doi:10.1111/j.1444-0938.20 11.00687.x

61. Imayasu M, Shiraishi A, Ohashi Y, Shimada S, Cavanagh HD. Effects of multipurpose solutions on corneal epithelial tight junctions. Eye Contact Lens. 2008;34(1):50-55. doi:10.1097/ICL.0b $013 \mathrm{e} 318073 \mathrm{cbdb}$

62. Santodomingo-Rubido J, Mori O, Kawaminami S. Cytotoxicity and antimicrobial activity of six multipurpose soft contact lens disinfecting solutions. Ophthalmic Physiol Opt. 2006;26(5):476-482. doi:10.1111/j.1475-1313.2006.00393.x

63. Buck SL, Rosenthal RA, Abshire RL. Amoebicidal activity of a preserved contact lens multipurpose disinfecting solution compared to a disinfection/neutralisation peroxide system. Cont Lens Anterior Eye. 1998;21(3):81-84. doi:10.1016/S1367-0484(98)80027-1

64. Rosenthal RA, McAnally CL, McNamee LS, Buck SL, Schlitzer RL, Stone RP. Broad spectrum antimicrobial activity of a new multi-purpose disinfecting solution. CLAO J. 2000;26(3):120-126.

65. Codling CE, Maillard J-Y, Russell AD. Aspects of the antimicrobial mechanisms of action of a polyquaternium and an amidoamine. J Antimicrob Chemother. 2003;51(5):1153-1158. doi:10.1093/jac/dkg228 
66. Rosenthal RA, Bell WM, Abshire R. Disinfecting action of a new multi-purpose disinfection solution for contact lenses. Cont Lens Anterior Eye. 1999;22(4):104-109. doi:10.1016/S1367-0484(99)80010-1

67. Matoba AY, Peterson JR, Wilhelmus KR. Dendritiform keratopathy associated with exposure to polyquarternium-1, a common ophthalmic preservative. Ophthalmology. 2016;123(3):451-456. doi:10. 1016/j.ophtha.2015.10.063

68. Callahan D, Kovacs C, Lynch S, Rah M. Biocidal efficacy of multipurpose solutions against gram-negative organisms associated with corneal infiltrative events. Clin Exp Optom. 2017;100(4):357-364. doi:10.1111/cxo.12509

69. Zimmerman AB, Emch AJ, Geldis J, Nixon GJ, Mitchell GL. Contact lens corneal inflammatory events in a University population. Optom Vis Sci. 2016;93(1):42-49. doi:10.1097/OPX.0000000000000746

70. Santodomingo-Rubido J. The comparative clinical performance of a new polyhexamethylene biguanide- vs a polyquad-based contact lens care regime with two silicone hydrogel contact lenses. Ophthalmic Physiol Opt. 2007;27(2):168-173. doi:10.1111/j.14751313.2006.00449.x

71. Berntsen DA, Hickson-Curran SB, Jones LW, et al. Subjective comfort and physiology with modern contact lens care products. Optom Vis Sci. 2016;93(8):809-819. doi:10.1097/OPX.0000000000000901

72. Lievens CW, Kannarr S, Zoota L, Lemp J. Lid Papillae improvement with hydrogen peroxide lens care solution use. Optom Vis Sci. 2016;93(8):933-942. doi:10.1097/OPX.0000000000000890

73. Guillon M, Maissa C, Wong S, Patel T, Garofalo R. The influence of lens care systems on eyelid tissue changes during silicone hydrogel contact lens wear. Cont Lens Anterior Eye. 2018;41(4):362-368. doi:10.1016/j.clae.2018.02.141

74. Dutot M, Warnet J-M, Baudouin C, Rat P. Cytotoxicity of contact lens multipurpose solutions: role of oxidative stress, mitochondrial activity and P2X7 cell death receptor activation. Eur J Pharm Sci. 2008;33(2):138-145. doi:10.1016/j.ejps.2007.10.006

75. Tilia D, Lazon de la Jara P, Weng R, Naduvilath T, Willcox MDP. Short-term clinical comparison of two dual-disinfection multipurpose disinfecting solutions. Eye Contact Lens. 2014;40(1):7-11.

76. Narayana BL, Rao P, Bhat S, Vidyalakshmi K. Comparison of the antimicrobial efficacy of various contact lens solutions to inhibit the growth of Pseudomonas aeruginosa and Staphylococcus aureus. Int J Microbiol. 2018;2018:5.

77. Reindel W, Merchea MM, Rah MJ, Zhang L. Meta-analysis of the ocular biocompatibility of a new multipurpose lens care system. Clin Ophthalmol. 2013;7:2051-2056.
78. Fears AC, Metzinger RC, Killeen SZ, Reimers RS, Roy CJ. Comparative in vitro effectiveness of a novel contact lens multipurpose solution on Acanthamoeba castellanii. $J$ Ophthalmic Inflamm Infect. 2018;8(1):19. doi:10.1186/s12348-018-0161-8

79. Nichols JJ, Chalmers RL, Dumbleton K, et al. The case for using hydrogen peroxide contact lens care solutions: a review. Eye Contact Lens. 2019;45(2):69-82. doi:10.1097/ICL.0000000000000542

80. Pinna A, Sechi LA, Zanetti S, et al. Bacillus cereus keratitis associated with contact lens wear1 1The authors have no financial interest in any material used in this study. Ophthalmology. 2001;108 (10):1830-1834.

81. Merchea M The realities of hydrogen peroxide lens care use \& misuse in today's marketplace; 2017. Available from: https://www. fda.gov/media/103874/download. Accessed June 17, 2021.

82. Rosenthal RA, Stein JM, McAnally CL, Schlech BA. A comparative study of the microbiologic effectiveness of chemical disinfectants and peroxide-neutralizer systems. CLAO J. 1995;21(2):99-110.

83. Yanai R, Yamada N, Ueda K, et al. Evaluation of povidone-iodine as a disinfectant solution for contact lenses: antimicrobial activity and cytotoxicity for corneal epithelial cells. Cont Lens Anterior Eye. 2006;29(2):85-91. doi:10.1016/j.clae.2006.02.006

84. Isenberg SJ. The ocular application of povidone-iodine. Community Eye Health. 2003;16(46):30-31.

85. Nakagawa M, Nakagawa R, Willcox MDP, Vijay AK. Effect of hygiene procedures on lens case contamination with povidone-iodine or multipurpose disinfecting solutions. Optom Vis Sci. 2021;98(6):563-569. doi:10.1097/OPX.0000000000001700

86. Kilvington S. Antimicrobial efficacy of a povidone iodine (PI) and a one-step hydrogen peroxide contact lens disinfection system. Cont Lens Anterior Eye. 2004;27(4):209-212. doi:10.1016/j.clae.2004. 09.001

87. Kobayashi T, Gibbon L, Mito T, Shiraishi A, Uno T, Ohashi Y. Efficacy of commercial soft contact lens disinfectant solutions against Acanthamoeba. Jpn J Ophthalmol. 2011;55(5):547-557. doi:10.1007/ s10384-011-0062-y

88. Yamasaki K, Saito F, Ota R, Kilvington S. Antimicrobial efficacy of a novel povidone iodine contact lens disinfection system. Cont Lens Anterior Eye. 2018;41(3):277-281. doi:10.1016/j.clae.2017.12.001
Clinical Optometry

\section{Publish your work in this journal}

Clinical Optometry is an international, peer-reviewed, open access journal publishing original research, basic science, clinical and epidemiological studies, reviews and evaluations on clinical optometry. All aspects of patient care are addressed within the journal as well as the practice of optometry including economic and business analyses. Basic and clinical research papers are published that cover

Submit your manuscript here: https://www.dovepress.com/clinical-optometry-journal all aspects of optics, refraction and its application to the theory and practice of optometry. The manuscript management system is completely online and includes a very quick and fair peer-review system, which is all easy to use. Visit http://www.dovepress.com/ testimonials.php to read real quotes from published authors. 